\title{
Surface Resistance Imaging with a Scanning Near-Field Microwave Microscope
}

\author{
D. E. Steinhauer, C. P. Vlahacos, S. K. Dutta, F. C. Wellstood, and Steven M. Anlage ${ }^{a)}$ \\ Center for Superconductivity Research, Department of Physics, University of \\ Maryland, College Park, MD 20742-4111
}

\begin{abstract}
We describe near-field imaging of sample sheet resistance via frequency shifts in a resonant coaxial scanning microwave microscope. The frequency shifts are related to local sample properties, such as surface resistance and dielectric constant. We use a feedback circuit to track a given resonant frequency, allowing measurements with a sensitivity to frequency shifts as small as two parts in $10^{6}$ for a $30 \mathrm{~ms}$ sampling time. The frequency shifts can be converted to sheet resistance based on a simple model of the system.
\end{abstract}

There is a growing need to develop non-destructive microscopy techniques to quantitatively measure the microwave properties of materials on a length scale much less than the free space wavelength. For example, spatially resolved measurements of complex conductivity would be of significant utility for evaluating oxide superconducting and ferroelectric thin film samples. Sensitivity to microwave and millimeter wave surface resistance and dielectric constant have been previously demonstrated; for example, Bryantand Gunnl used a coaxia bresonator to measure semicon-

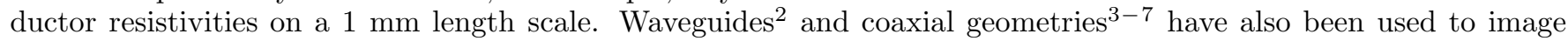
conductivity and dielectric constant contrast. In this letter, we describe the use of a microwave microscope with an open-ended coaxial probe to quantitatively map the surface resistance of a metallic thin film.

The key element in our system 1 is a 2 meter-long resonant coaxial transmission line (see Fig. 1). One end of the line is connected to an open-ended coaxial probe and the other end is weakly coupled to a microwave source via a capacitor $C_{D}$. Near-field microwave energy at the exposed tip of the probe center conductor is coupled to the sample. As the sample is scanned beneath the probe tip, the resonant frequencies and quality factor $\mathrm{Q}$ of the open transmissionline shift depending on the surface properties of the region of the sample closest to the probe's center conductor 0.8 We measure the microwave power reflected back up the transmission line with a diode detector 9 By using a fixed frequency source near one of the resopances $f_{R}$, and scanning a sample underneath the probe, one can map the reflected power and generate an image. 6 However, this results in a convolution of two distinct contrast mechanisms: the frequency shift of the standing wave resonances and the change in $\mathrm{Q}$.

To disentangle these effects, we have developed a frequency-following feedback circuit, as shown in Fig. 1. We frequency modulate the source at a rate $f_{F M} \approx 3 \mathrm{kHz}$ with a deviation of about $\pm 3 \mathrm{MHz}$ and use a feedback loop to keep the average microwave source frequency locked to a specific resonant frequency $f_{R}(t)$ of the microscope. To accomplish this, the diode detector output voltage is amplified and sent to a lock-in amplifier referenced at the frequency $f_{F M}$. The lock-in output is time-integrated; this voltage signal $V_{o u t}$ is added to the $f_{F M}$ oscillator signal

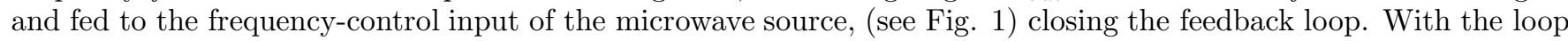
locked, the signal $V_{\text {out }}$ is proportional to the frequency shift. We use a computer to record $V_{\text {out }}$, while controlling the sample position using a two-axis translation stage. This data can be compiled into a gray-scale frequency shift image.

One advantage to this circuit is its speed; the frequency shift can be recorded while the sample is in motion beneath the probe. We typically sample at a rate of $30 \mathrm{~Hz}$, so that a $1 \times 1 \mathrm{~cm}$ sample can be scanned with a resolution of 100 $\mu \mathrm{m}$ in about 5 minutes.

As shown in the inset to Fig. 1, we modell the interaction between the probe and sample as a capacitance $C_{X}$ between the center conductor of the probe and the sample, and a resistance $R_{X}$ connected to the outer conductor via a second capacitor $C_{O}$. The capacitance $C_{X}$ is determined by the area of the probe center conductor and the height of the probe above the sample. Because of the large area of the outer conductor, we take $C_{O} \gg C_{X}$; in this approximation, $R_{X}$ is connected directly to the grounded outer conductor. For simplicity, we use a parallet-plate approximation for $C_{X}$, and assume that $R_{X}$ is equivalent to the microwave surface resistance of the sample 10 . 11 . To model the system we also include the decoupler capacitance $\left(C_{D} \approx 0.17 \mathrm{pF}\right)$ and attenuation in the transmission line 12 We calculate shifts in the resonant frequencies as a function of the above parameters using standard microwave circuit theory.13

We tested the system by measuring frequency shift as a function of sheet resistance $R_{X}$. To vary $R_{X}$, we used a variable-thickness thin-film oxidized aluminum sample. The aluminum film was deposited on a glass substrate by

a) Electronic mail: anlage@squid.umd.edu; color versions of the figures in this paper are available at http://www.csr.umd.edu 
thermal evaporation. The glass and the source were arranged so that the source was much closer to one side of the substrate than the other, producing a smooth variation in the thickness of the film. After oxidation in air, the thickness of the conductive aluminum is less than $6 \mathrm{~nm}$, and much less than the skin depth at microwave frequencies, so that there is essentially no reactive impedance presented by the sample 10 Using two-point resistance measurements of constant-thickness strips of the sample, we measured $R_{X}$ values which ranged smoothly from $58 \Omega / \square$ to $42 \mathrm{k} \Omega / \square$ across the sample.

To measure the frequency shift, a probe with a $480 \mu \mathrm{m}$ diameter center conductor was positioned at various heights above the sample, and a microscope resonance at $7.8 \mathrm{GHz}$ was chosen. The data points in Fig. 2a indicate measurements at different heights above the sample. For decreasing $R_{X}$ at a fixed height, the resonant frequencies shift downward. This can be understood as follows: as $R_{X}$ decreases, the boundary condition of the resonator tends from an open circuit toward a short circuit, changing the circuit from a half-wave resonator toward a quarter-wave resonator, and lowering the resonant frequency. We note that the graph of frequency shift vs. $R_{X}$ flattens out for low $R_{X}$, indicating reduced sensitivity to sheet resistance in this region. In addition, the curves for smaller heights have steeper slopes, demonstrating that the sensitivity to $R_{X}$ increases as the probe-sample separation is reduced.

For comparison, the expected curves from a microwave analysis of the circuit are shown as lines in Fig. 2a. For $R_{X}<377 \Omega / \sqrt{\epsilon_{r}}$, where $\epsilon_{r}$ is the dielectric constant of the glass substrate, the aluminum conductance dominates; in this region, we ignored the presence of the substrate.10 Because of the large diameter of the probe, there is an uncertainty in the height of the center conductor relative to the sample. Accordingly, we fitted the height for one data set (the $38 \mu \mathrm{m}$ set) and then used the measured heights relative to this set for the other data sets. For low $R_{X}$, the $38 \mu \mathrm{m}$ fit agrees quite well with our experimental data, while the curves at 88 and $188 \mu \mathrm{m}$ don't agree as well; this discrepancy may be due to a breakdown of the parallel plate approximation as the sample-probe separation approaches the radius of the probe center conductor.

We also tested the frequency shift as a function of height for various values of $R_{X}$ (see Fig. 2 $\mathrm{b}$ ). Notice that as the probe is moved closer to the sample, the resonant frequencies drop. This is expected since the increase in coupling capacitance between the probe and sample effectively increases the length of the resonant circuit, which decreases the resonant frequencies. 7 As the lines in Fig. $2 \mathrm{~b}$ show, the model agrees with our experimental data for low values of $R_{X}$ where the effect of the glass substrate can be safely ignored, and for heights small compared to the radius of the probe center conductor, where the parallel plate approximation is valid.

Our simple model doesn't take into account the presence of the glass substrate (a dielectric). For high $R_{X}$, if the glass were not present, the resonant frequency shifts would tend to zero (defined as the resonant frequency when the probe is $>1 \mathrm{~mm}$ from the sample). This is not the case, as shown by the experimental data in Fig. 2a. As shown in the inset to Fig. 2b b we empirically model the effect of the glass by taking $R_{X}=0$, and modeling $C_{X}$ as two capacitors in series, $\frac{1}{C_{X}}=\frac{1}{C^{\prime}}+\frac{1}{C(h)}$, with an air gap capacitance $C(h)=\frac{\epsilon_{0} A^{\prime}}{h}$. The two fitting parameters are $A^{\prime}$, an effective area for the probe, and the fringe capacitance $C^{\prime}$ through the substrate. For the glass substrate the best fit to our model gave $A^{\prime}=0.65 A$ and $C^{\prime}=0.044 \mathrm{pF}$, where $A$ is the area of the probe's center. We used this modified model for plotting frequency shifts for high $R_{X}$ in Fig. 2aa, and for plotting the model curve for glass in Fig. 2pb. In principle, the empirical values of $A^{\prime}$ and $C^{\prime}$ could be used to determine the dielectric constant of a material.

To demonstrate the imaging capabilities of the system, we imaged a 3.2 lines $/ \mathrm{mm}$ NIST standard resolution target consisting of a patterned chromium thin film on glass (see Fig. 3a). Figure 3b shows a frequency shift image of the resolution target. As shown in the figure, the resonant frequencies shifted downward by about $300 \mathrm{kHz}$ when the probe moved from over glass to over a chromium line. This is less than we would expect for a uniform film with a sheet resistance of $10 \Omega / \square$, suggesting that the small width of the chromium lines $(156 \mu \mathrm{m})$ relative to the probe outer conductor diameter $(860 \mu \mathrm{m})$ reduces the magnitude of the observed frequency shifts from that expected for a uniform film. We note that the $300 \mu \mathrm{m}$-wide decimal point in " 3.2 " is visible, even though it is smaller than the probe outer conductor diameter; however, the decimal point appears dimmer than the chromium lines and is doughnut-shaped, also suggesting that the film pattern geometry has an additional effect on the magnitude of the frequency shifts. Because of the concentration of the fields near the inner conductor tip, the spatial resolution is determined by the diameter of the inner conductor.t

By observing circuit output noise, we find that with a sampling time of $30 \mathrm{~ms}$ the frequency-following circuit is sensitive to frequency shifts as small as two parts in $10^{6}$. Given the sensitivity to frequency shift and the data in Fig. 2a, we can estimate the sensitivity to changes in $R_{X}$. For our $480 \mu \mathrm{m}$ center conductor probe at a height of $38 \mu \mathrm{m}$, we find $\Delta R_{X} / R_{X}=5 \times 10^{-2}$ for $R_{X}=100 \Omega / \square$. To achieve finer spatial resolution and maintain the same frequency shift sensitivity to $R_{X}$, one could decrease the probe inner conductor diameter, and decrease the probesample separation $(h)$, in order to maintain the same probe-sample capacitance. One limitation of this technique is that changes in the probe-sample separation $(\Delta h)$ must remain small relative to $h$ throughout the scan. If the sample is not planar, changes in probe-sample capacitance will dominate at small $h$, thus setting a lower limit to how small $h$ can be for quantitative surface resistance imaging. A solution to this problem would be an active feedback system 
to keep the probe-sample separation constant 14

In conclusion, we have demonstrated the use of a frequency-following circuit for microwave imaging of the surface resistance of metallic samples. The sensitivity to surface resistance depends primarily on the probe-sample capacitance. Our current system has the best surface resistance sensitivity in the range from about 20 to $200 \Omega / \square$. In the low surface resistance range, $R_{X} \lesssim 150 \Omega / \square$, our results on a uniform sample show reasonable agreement with a simple model based on standard microwave circuit theory.

This work has been supported by NSF-MRSEC grant \# DMR-9632521, NSF grant \# ECS-9632811, one of us (S.M.A.) by NSF NYI grant \# DMR-9258183, and by the Center for Superconductivity Research.

${ }^{1}$ C. A. Bryant and J. B. Gunn, Rev. Sci. Instr. 36, 1614 (1965).

${ }^{2}$ M. Golosovsky, A. Galkin and D. Davidov, IEEE Trans. Microwave Theor. Tech., 44 (7), 1390 (1996).

${ }^{3}$ S. J. Stranick, L. A. Bumm, M. M. Kamna and P. S. Weiss, in Photons and Local Probes, eds. O. Marti and R. Möller, (Kluwer, Netherlands, 1995), p. 221.

4 T. Wei and X.-D. Xiang, Appl. Phys. Lett. 68 (24), 3506 (1996).

${ }^{5}$ F. Keilmann, D. W. van der Weide, T. Eickelkamp, R. Merz and D. Stöckle, Opt. Commun., 129, 15 (1996).

${ }^{6}$ B. Knoll, F. Keilmann, A. Kramer and R. Guckenberger, Appl. Phys. Lett. 70 (20), 2667 (1997).

${ }^{7}$ C. P. Vlahacos, R. C. Black, S. M. Anlage, A. Amar and F. C. Wellstood, Appl. Phys. Lett. 69 (21), 3274 (1996), and references therein.

${ }^{8}$ S. M. Anlage, C. P. Vlahacos, Sudeep Dutta and F. C. Wellstood, IEEE Trans. Appl. Supercond. 7 (3), 3686 (1997).

${ }^{9}$ Hewlett-Packard Company, Santa Clara, California: microwave diode detector, part \# $8473 \mathrm{C}$.

${ }^{10}$ N. Klein, H. Chaloupka, G. Müller, S. Orbach, H. Piel, B. Roas, L. Schultz, U. Klein and M. Peiniger, J. Appl. Phys. 67 (11), 6940 (1990).

11 J. C. Booth, Dong Ho Wu and Steven M. Anlage, Rev. Sci. Instrum. 65 (6), 2082 (1994).

12 Private communication with W. L. Gore and Assoc., Inc.: for ReadyFlex cable, attenuation constant $\alpha=0.333 \mathrm{~dB} / \mathrm{ft}$ at 7.8 $\mathrm{GHz}$; dielectric constant $\epsilon_{r}=1.15$; characteristic impedence $Z_{0}=50 \Omega$.

${ }^{13}$ S. Ramo, J. R. Winnery and T. Van Duzer, Fields and Waves in Communication Electronics, 3rd. ed. (Wiley, New York, 1994), Chap. 5.

14 D. Rugar, H. J. Mamin and P. Guethner, Appl. Phys. Lett. 55 (25), 2588 (1989). 


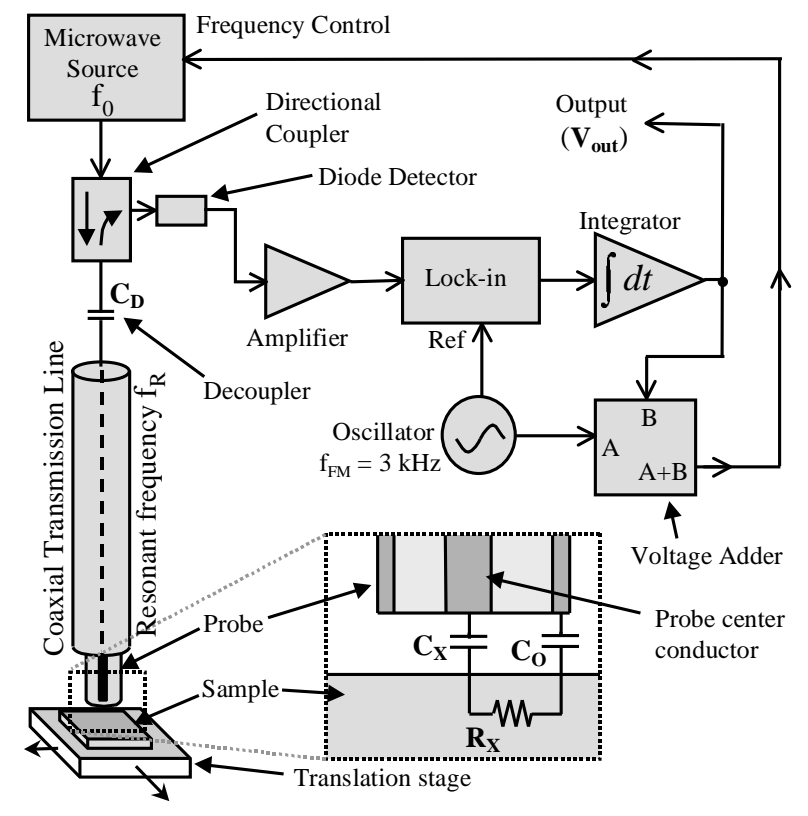

FIG. 1. Schematic of the near-field scanning microwave microscope. The inset shows the probe tip and sample, with the probe-sample capacitance $\mathrm{C}_{X}$ and the sample resistance $\mathrm{R}_{X}$. The feedback circuit keeps the microwave source locked onto a resonant frequency of the circuit consisting of a coaxial transmission line and open-ended coaxial probe. 
(a)

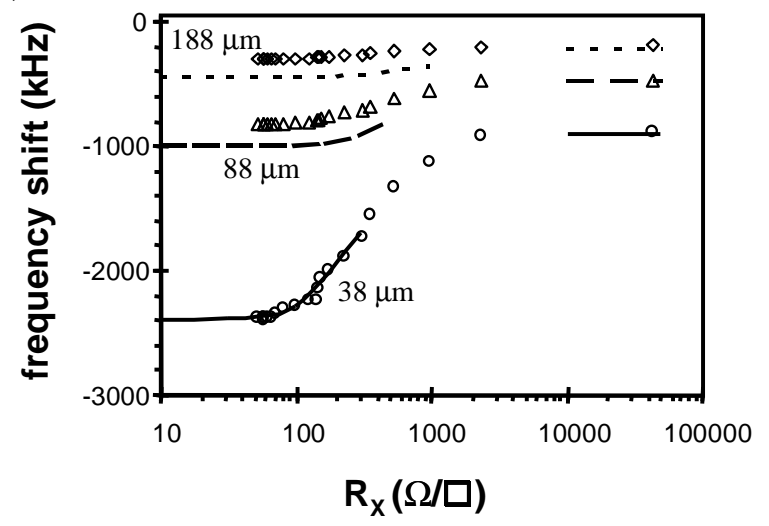

(b)

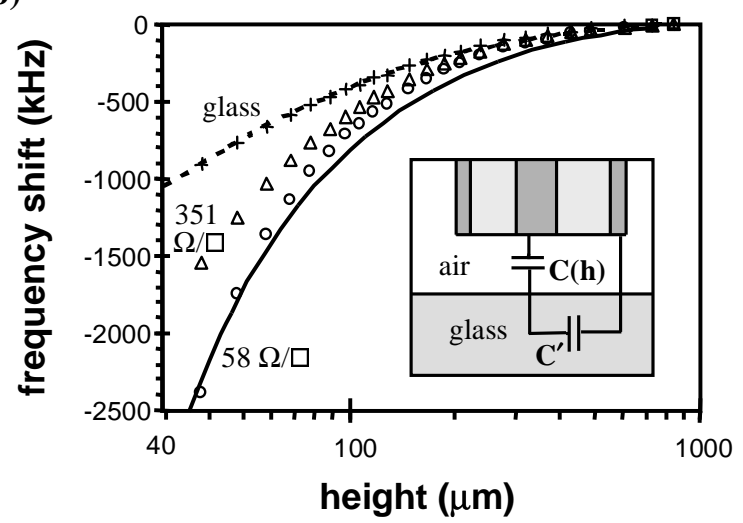

FIG. 2. The symbols show the measured frequency shift vs. (a) surface resistance $\mathrm{R}_{X}$, and (b) probe-sample separation. The lines indicate expected frequency shift based on microwave circuit analysis of the system. A probe with a $480 \mu \mathrm{m}$ diameter center conductor was used, at a frequency of $7.8 \mathrm{GHz}$. The inset is a diagram of our model for the interaction between the probe and the sample when no film is present on top of the glass substrate. 
(a)

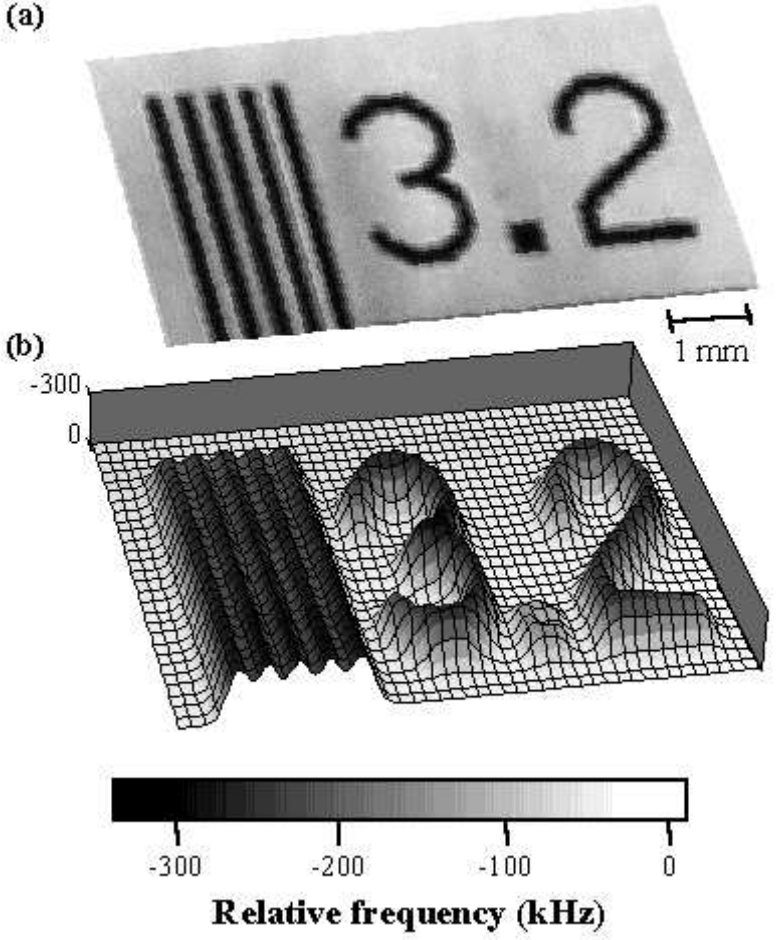

FIG. 3. (a) Optical photograph of a 3.2 lines $/ \mathrm{mm}$ resolution target. The target consists of a pattern of chromium thin film lines on glass. (b) Frequency shift surface plot of the resolution target. A probe with a $200 \mu$ m diameter center conductor was used at a height of $5 \mu \mathrm{m}$ and a frequency of $10.7 \mathrm{GHz}$. The frequency shifts are relative to the resonant frequency when the probe is over glass. 
This figure "figure1.gif" is available in "gif" format from: http://arXiv.org/ps/cond-mat/9712142v1 
This figure "figure2.gif" is available in "gif" format from: http://arXiv.org/ps/cond-mat/9712142v1 
This figure "figure3.gif" is available in "gif" format from: http://arXiv.org/ps/cond-mat/9712142v1 